\title{
Self-Guided Clinical Cases for Medical Students Based on Postmortem CT Scans of Cadavers
}

\author{
MICHAEL BOHL, ${ }^{*}$ WEBSTER FRANCOIS, AND THOMAS GEST \\ Division of Anatomical Sciences, University of Michigan Medical School, Ann Arbor, Michigan
}

\begin{abstract}
In the summer of 2009, we began full body computed tomography (CT) scanning of the pre-embalmed cadavers in the University of Michigan Medical School (UMMS) dissection lab. We theorized that implementing web-based, self-guided clinical cases based on postmortem CT (PMCT) scans would result in increased student appreciation for the clinical relevance of anatomy, increased knowledge of cross-sectional anatomy, and increased ability to identify common pathologies on CT scans. The PMCT scan of each cadaver was produced as a DICOM dataset, and then converted into a Quicktime movie file using Osirix software. Clinical cases were researched and written by the authors, and consist of at least one Quicktime movie of a PMCT scan surrounded by a novel navigation interface. To assess the value of these clinical cases we surveyed medical students at UMMS who are currently using the clinical cases in their coursework. Students felt the clinical cases increased the clinical relevance of anatomy (mean response 7.77/10), increased their confidence finding anatomical structures on CT (7.00/10), and increased their confidence recognizing common pathologies on CT (6.17/10). Students also felt these clinical cases helped them synthesize material from numerous courses into an overall picture of a given disease process $(7.01 / 10)$. These results support the conclusion that our clinical cases help to show students why the anatomy they are learning is foundational to their other coursework. We would recommend the use of similar clinical cases to any medical school utilizing cadaver dissection as a primary teaching method in anatomy education. Clin. Anat. 24:655-663, 2011. @ 2011 Wiley-Liss, Inc.
\end{abstract}

Key words: novel techniques in medical education; radiology in anatomy education; clinical relevance in anatomy education; teaching cross-sectional anatomy

\section{INTRODUCTION}

Since the late 1960 s, radiological images have been used to supplement gross anatomy education for undergraduate medical students (Stassa and Evans, 1969; Forrester, 1971). Reports from the last 25 years on medical student and anatomy faculty impressions of supplementing cadaver dissection with radiological images have been resoundingly positive, with claims that radiology makes anatomy more clinically relevant and stimulates greater interaction between anatomy students and faculty (McNiesh et al., 1983; Pantoja et al., 1985; Tavares et al., 2000; Miles, 2005; Turmezei et al., 2009). Studies have further shown that using radiological images in cadaver dissection improves students' short and long-term ability to identify anatomic

\footnotetext{
*Correspondence to: Michael A. Bohl, Division of Anatomical Sciences, University of Michigan Medical School, Ann Arbor, Michigan 48109, USA. E-mail: mbohl@umich.edu
}

Received 14 November 2010; Revised 10 January 2011; Accepted 12 January 2011

Published online 8 March 2011 in Wiley Online Library (wileyonlinelibrary.com). DOI 10.1002/ca.21143 
structures in diagnostic radiographs (Erkonen et al. 1990, 1992a,b), a skill that Jastrow and Vollrath (2003) argue is essential to the training of medical students and doctors. As Khalil et al. (2005) explain, the majority of medical students will not become radiologists, and so they must develop a fundamental knowledge of radiological imaging if they are to competently understand the consultation reports they receive from radiologists. It has been shown that one effective way of imparting this competency in reading radiological imaging, specifically computed tomography (CT) scans, is to incorporate radiology into first-year medical student anatomy courses (Tavares et al., 2000; de Barros et al., 2001). Chew et al. (2006) explain that combining postmortem cadaver CT (PMCT) scans with anatomical dissection will not only enhance the learning of anatomical concepts, but the use of the scans themselves could improve students' overall understanding of diagnostic radiology in medicine.

Some problems have been cited regarding the use of PMCT scans in anatomy education. One problem is getting students to overcome their lack of confidence when viewing radiological images. Our personal experience is that one of the most common complaints from students regarding the use of radiology in anatomy education is that they feel they do not know enough anatomy yet to understand what they are seeing in the radiological images. This becomes a circular argument then as professors tell students that to learn the anatomy they must look at the radiography. Unless the radiological images are clearly marked with identifying labels, students often give up studying the images, feeling overwhelmed by radiology rather than empowered by it. We sought to overcome this early lack of confidence by implementing a novel navigation interface with the PMCT scans. This interface directs students to various findings on the PMCT scan without actually highlighting any structures. Students are therefore encouraged to explore the scans and try to interpret the threedimensional anatomy by themselves, while at the same time providing them a guide so that they can be reasonably sure they are seeing what we intend them to see. Another problem with PMCT scans was demonstrated by Chew et al. (2006), who concluded that post-embalming CT scans show significant differences from CT scans of living patients, due in large part to artifacts of preservation. This could likely have a negative effect on the utility of PMCT scan in anatomy education due to the degraded resolution of soft tissue anatomy. Although two recent studies that provided PMCT scans of embalmed cadavers to medical students have reported positive results with their scans (Jacobson et al., 2009; Lufler et al., 2010), we hope to enhance the quality and educational utility of our PMCT scans by obtaining them prior to embalming. This way we can be sure that the scans we are presenting to medical students resemble those of living patients as accurately as possible.

Recent attempts to provide students with PMCT scans of cadavers have proven to be successful. Lufler et al. (2010) provided a class of 179 students with four PMCT scans of cadavers under dissection, and conducted a quantitative assessment of the effect on test score that these PMCT scans had. They found that "use of [PMCT] scans in medical gross anatomy is predictive of performance in [their] course and on questions requiring knowledge of anatomical spatial relationships." Jacobson et al. (2009) intended to integrate basic and clinical science teaching by using PMCT scans along with living patient images to create virtual patients. Their student feedback was very positive and has encouraged them to continue using virtual patients in anatomy education. Similarly, we have developed a collection of web-based, self-guided clinical cases that lead anatomy students through interesting findings and common pathologies on the full-body CT scans of their donors. With these clinical cases, we sought not only to enhance the clinical relevance of anatomy education by exposing students to diagnostic radiology, but also to synthesize material learned in numerous different classes into overall pictures of the pathological, physiological, anatomical, and radiological presentation of common diseases. By including images of gross pathology, histology, and discussions on pathophysiology alongside interactive CT scans of students' donors, we intended to increase the value of our clinical cases to students, thereby encouraging greater student use of this resource. Considering the potential value that PMCT imaging has for anatomy education, we believe that implementing web-based, self-guided clinical cases based on PMCT scans in first-year medical anatomy education will result in increased student appreciation for the clinical relevance of anatomy, increased knowledge of crosssectional anatomy, and increased ability to identify common pathological processes on CT scans.

\section{METHODS}

Beginning in the summer of 2009, we established a procedure for creating full-body, pre-embalming PMCT scans of every cadaver in the medical school dissection lab. Through a gratis arrangement with the UMMS Department of Radiology, scanners were made available for our use at 4:00 AM. The PMCT scan of each cadaver was produced as a DICOM dataset, and then converted into a Quicktime movie file using Osirix software. Thirty cadavers are used in the dissection lab for first-year medical students, and all thirty cadavers were scanned in both 2009 and 2010. Because of the unforeseen complications, only 21 PMCT datasets were available for creating clinical cases in 2010.

Clinical cases were researched and written by the authors. Each clinical case consists of text and pictures that describe the normal and pathological findings of a given disease process on gross, histologic, physiologic, and/or biochemical examination. Each clinical case also contains radiological imaging including at least one Quicktime movie of a PMCT scan surrounded by our novel navigation interface. This interface consists of three scales: one scale below that is the movie file's time scale and corre- 
lates to the level of cross-section, and two scales on the left and upper side of the CT image which are used to create an alphanumeric grid that enables students to identify specific points on the CT image. Above and below the CT image is text containing directions for locating interesting findings in the CT scan and explanations of the clinical and anatomical significance of these findings. See Figure 1 for an example of a clinical case and the CT navigation grid. We currently have 22 clinical cases written and available for student use on the UMMS anatomy website. The available cases are listed below:

1. Abdominal aortic aneurysm

2. Alzheimer's disease

3. Atherosclerosis

4. Cardiomegaly

5. Cardiovascular anatomy (normal)

6. Carotid endarterectomy

7. Cervical corpectomy

8. Cholelithiasis

9. Hiatal hernia

10. Hip replacement

11. Laminectomy

12. Nephrolithiasis

13. Osteophytosis

14. Pacemaker

15. Pericardial effusion

16. Pleural effusion

17. Portacath

18. Renal cyst

19. Scoliosis

20. Starry spleen

21. Stroke

22. Vesicocutaneous fistula

To assess the value of these clinical cases prior to their use by first-year medical students, we made the cases available online to second, third, and fourth year medical students at UMMS and asked these students to view the cases and provide us with written feedback. We used the feedback gained from these students to help us determine what changes, if any, to make to the clinical cases prior to their use by the first-year students.

Use of the clinical cases was voluntary for the firstyear medical students. The existence and utility of the clinical cases were presented to the students in one of their first anatomy lectures of the year, and though students were made aware that no test questions were going to come directly from the clinical cases, they were reminded that the material presented in the clinical cases would be material that they would see on their examinations. We encouraged students to view the clinical cases before, during, and after dissection laboratory sessions, especially if their assigned body demonstrated a finding on CT that might soon be uncovered by dissection. We also periodically reminded students of clinical cases that pertained to their current focus of dissection (for example, during dissection of the deep back we sent the students reminders to look at the clinical cases for scoliosis, laminectomy, and osteophytosis). Because the clinical cases are accessed via a publically accessible portion of the UMMS anatomy website and student use of the clinical cases is not mandated, we were not able to collect log data and other details of how students used the clinical cases. Our only measure of student use of the clinical cases was via student participation in the survey described below.

Three months into the first-year curriculum, after the students had finished dissecting the superficial and deep back, the pectoral region, and the thoracic cavity, we sent a survey to all the first-year students soliciting their opinions of the CT-based clinical cases. The survey was created and the data analyzed using Qualtrics survey software (version 15853, Qualtrics Labs, Provo, UT). The following questions were asked on the survey (these question numbers correspond to those in Table 1 ):

1. How easy was it finding structures on the CT scans using the time and location grid?

2. Do you feel more confident in your ability to recognize anatomical structures on cross-sectional CT scan after reviewing these clinical cases?

3. Do you feel more confident in your ability to recognize common pathologies on CT scan after reviewing these clinical cases?

4. Do you think the CT-based clinical cases increase the clinical relevance of the anatomy taught in the M1 year?

5. Do the CT-based clinical cases help you to synthesize material you receive in numerous different classes (i.e., pathology, physiology, anatomy) into an overall picture of a disease presentation in a patient, including diagnosis via CT?

6. Do you think these CT-based clinical cases should be included as a permanent, testable part of M1 anatomy?

Space was left at the end of the survey for any comments or suggestions on improvement of the clinical cases. All questions were scored on a 1-10 scale $(1=$ definitely no or extremely difficult, $10=$ definitely yes or extremely easy). Student participation in the survey was voluntary and anonymous.

\section{RESULTS \\ Senior Student Feedback}

Feedback from second, third, and fourth year medical students was solicited for 2 weeks in the summer of 2010. Twenty-three students agreed to review the clinical cases and provide feedback. We received written comments from nine students, and incorporated their suggestions into the clinical cases prior to the beginning of the new medical school year (Fall 2010). Senior student feedback was very positive. One student wrote, "They're great... I think these cases could really reinforce what students learn in anatomy, and make the information more fun." Another student thought that "functionally [these clinical cases] are excellent, and would be an amazing way to learn radiology." A third-year medical student commented, "When I was taking anatomy it was very difficult for me to realize the clinical relevance of all the labs. It is only this year (my third year) that I realize why cer- 


\section{Clinical Cases Pericardial Effusion}

\section{Pericardial Effusion}

Similar to how a pleural effusion is an abnormal accumulation of fluid in the pleural space around the lungs, a pericardial effusion is an abnormal accumulation of fluid in the pericardial sac surrounding the heart. The fluid of a pericardial effusion can be number of different things:

1. Transudative - A transudate is an extravascular fluid that is low in protein, is generally acellular, and occurs in a non-inflammatory setting. It results from either an increase in hydrostatic pressure or a decrease in osmotic pressure inside blood vessels. A transudative pericardial effusion could occur in the setting of $\mathrm{CHF}$ (congestive heart failure-increased hydrostatic pressure in venous system as heart begins failing to push blood into the arterial system) or nephrotic syndrome, in which the kidneys leak massive amounts of protein resulting in hypoalbuminemia and so decreased osmotic pressure in the blood vessels.

2. Exudative - In comparison to a transudate, an exudate is an extravascular fluid that is seen in a setting of inflammation. There are many types of exudates with varied compositions. A pericardial effusion caused by an exudate is called a pericardial empyema, and most commonly occurs in the setting of infection.

3. Hemorrhagic - When blood leaks into and fills the pericardial sac, it is called a hemopericardium. Most commonly this is caused by trauma, but it can also be caused Hemorrhagic - When blood leaks into and fills the pericardial sac, it is called a hemopericardium. Most commonly this is caused by trauma, but it can also be caused
by retrograde dissection of the ascending aorta. An aortic dissection is when the tunica intima of the aorta tears, allowing blood to flow into the tunica media and split the media between the internal and external elastic laminae. This separation of the aortic wall, or aortic dissection, can move anterograde toward the iliac bifurcation or retrograde toward the aortic root. If the dissection occurs in the ascending aorta and moves retrograde, it can eventually lead to rupture of the aortic wall inside the pericardial sac, creating a massive hemopericardium.

4. Malignancy - A fluid secreting malignancy could also be the cause of a pericardial effusion.
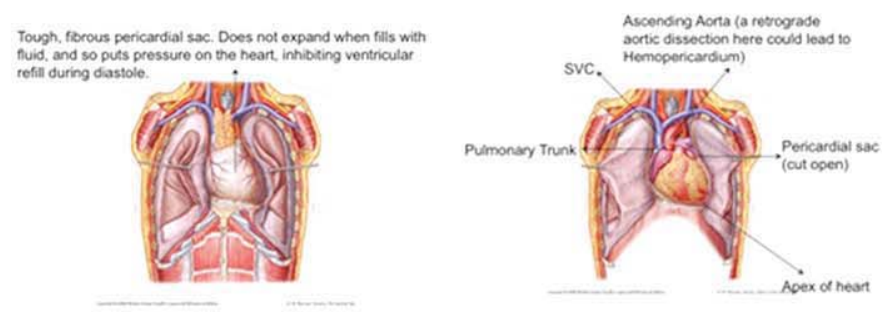

\section{Cardiac Tamponade}

The pericardial sac is a tough, fibrous structure that normally holds about 25-50 cc of fluid around the heart. As fluid begins building up to an abnormally high level in the pericardial sac, the sac resists stretching and so puts pressure in the heart After 150-250 cc of fluid has accumulated, a condition known as cardiac tamponade occurs. Cardiac tamponade is an emergency medical condition in which the fluid build-up in the pericardial sac exerts enough pressure on the heart to prevent refilling of the ventricles during diastole. This results in low stroke volume, low cardiac output, and eventually shock as the body enters a hypoperfusional state. Symptoms of cardiac tamponade are similar to those seen in shock. Classic cardiac tamponade presents 3 signs known as Beck's triad:

1. Hypotension - due to decreased stroke volume

2. Jugular venous distension - due to reduced venous return to the heart

3. Muffled heart sounds - due to the muffling effect of fluid surrounding the heart

\section{Pericardial Effusion on CT}

The image below is called a CTA (CT Angiography) scan, and is created by taking a CT scan of someone after a contrast dye has been injected into their circulatory system. Notice in this scan how blood containing the contrast appears as radiopaque as bone. As you can see, the heart in this patient is surrounded by a halo of fluid. The black space seen between the heart and the effusion is pericardial fat lying on the surface of the heart.

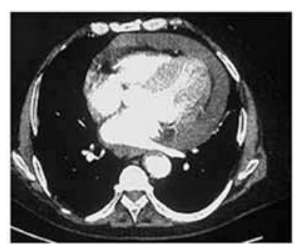

The movie below is the CT scan of cadaver 33492. Begin looking at the scan by familiarizing yourself with the heart, using the ascending aorta (time $=53, \mathrm{~K} 9$, very collapsed) to find the left ventricle, the SVC (time $=50, \mathrm{~J} 10$ ) to find the right atrium, the pulmonary trunk (time $=52$, L9) to find the right ventricle, and the pulmonary veins (time $=56, \mathrm{~J} 11$ and M12) to find the left atrium. Now look at the heart from time 50-60, noting how its chambers are surrounded first by a ring of black (normal pericardial fat) and then by a thick ring of gray. This thick outer ring of gray is all fluid that has accumulated in the pericardial sac. It is most prevalent around the left ventricle at time= $55-60, \mathrm{~N} 9$

This patient's pericardial effusion has forced other structures out of their normal orientation in the body. For example, notice how the trachea (time $=45-50$, K9) deviates to the right once inside the chest. This is due to the enlarged heart crowding into the superior mediastinum and pushing the trachea away from midline. Also notice how the right kidney (comes into view at time $=65,112$ ) is seen to be higher in the body than the left kidney (comes into view at time $=67, \mathrm{~N} 12$ ). Normally, the liver pushes the right kidney down below the level of the left kidney, but in this patient the greatly enlarged heart is forcing the left kidney below the level of the right kidney.

Fig. 1. Example of a clinical case-pericardial effusion. [Color figure can be viewed in the online issue, which is available at wileyonlinelibrary.com.] 


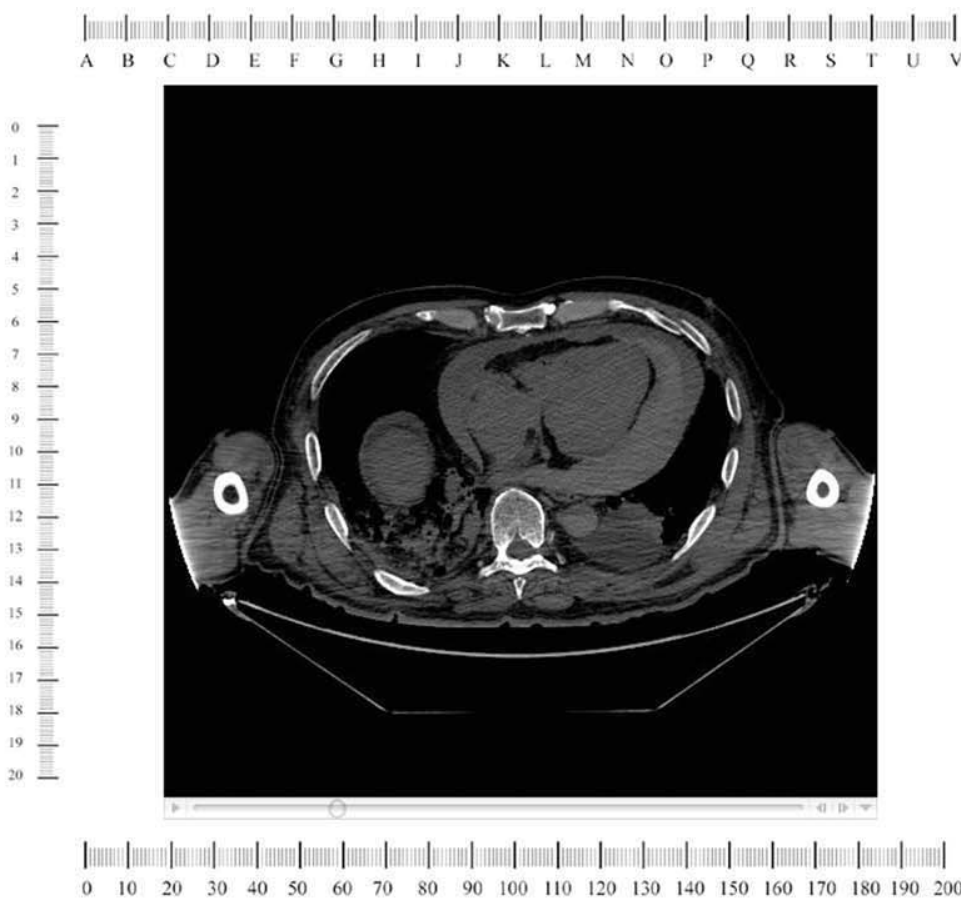

\section{Treatment}

Caring for someone with a pericardial effusion that is causing cardiac tamponade requires the support resources of a hospital. The patient's shock is managed until a procedure can be performed to remove the fluid from around the heart (i.e. giving oxygen to the patient and vasopressors to combat the systemic vasodilation and subsequent hypotension that is characteristic of shock). Removal of the fluid in the pericardial sac is done by pericardiocentesis. This procedure involves inserting a needle, usually under ultrasound guidance, into the pericardial sac and aspirating fluid from the sac. As you can see in the image below, the best place to insert the needle for a pericardiocentesis is along the left sternal border in the 5 th intercostal space. The cardiac notch of the left lung creates a bare area of the pericardial sac at this point, giving direct access to the heart. Not only is the pericardial sac unobstructed by the lung at this point, but it is also near the apex of the heart, where fluid is most likely to accumulate.

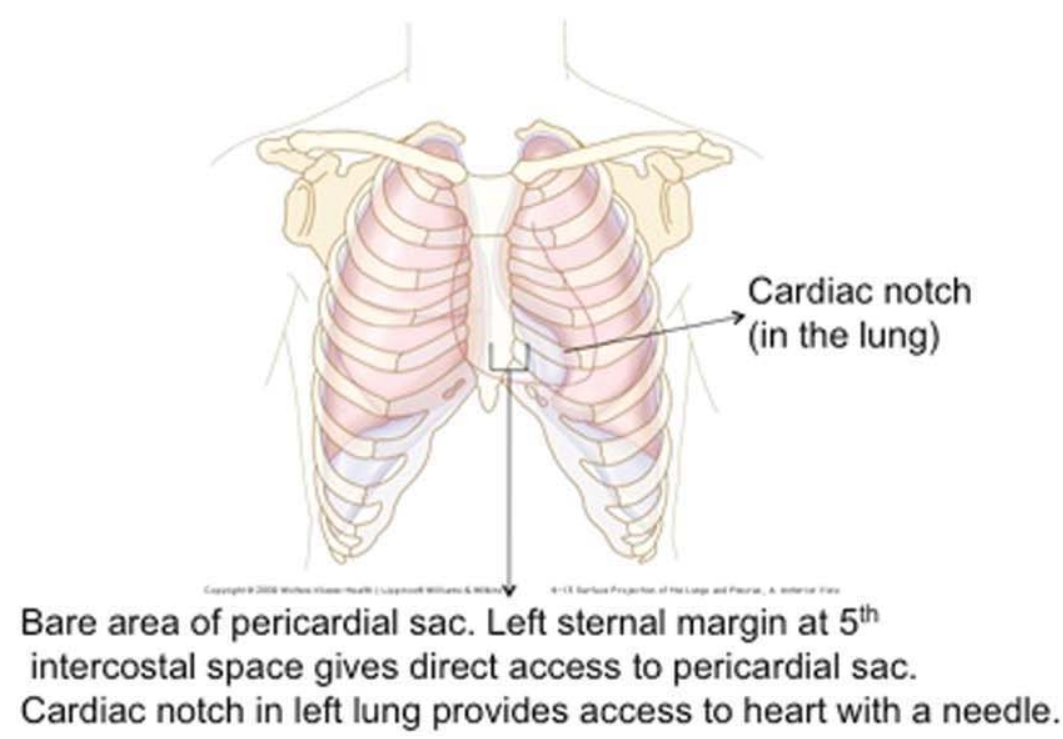

Fig. 1. Continued

tain structures are so important to memorize. I think that adding these CT scans, along with the text, is a great way to show [first-year students] that the info from anatomy labs is actually used in the 'real world' and that it is something that they can even visualize on imaging. I would have really loved to have this resource when I was a [first-year student]!" Another student thought the cases were "very well organized and easy to follow" and they "enjoyed the inclusion of histopathology images and tie-ins." After receiving 
TABLE 1. Mean Respondent Scores [Color table can be viewed in the online issue, which is available at wileyonlinelibrary.com.]

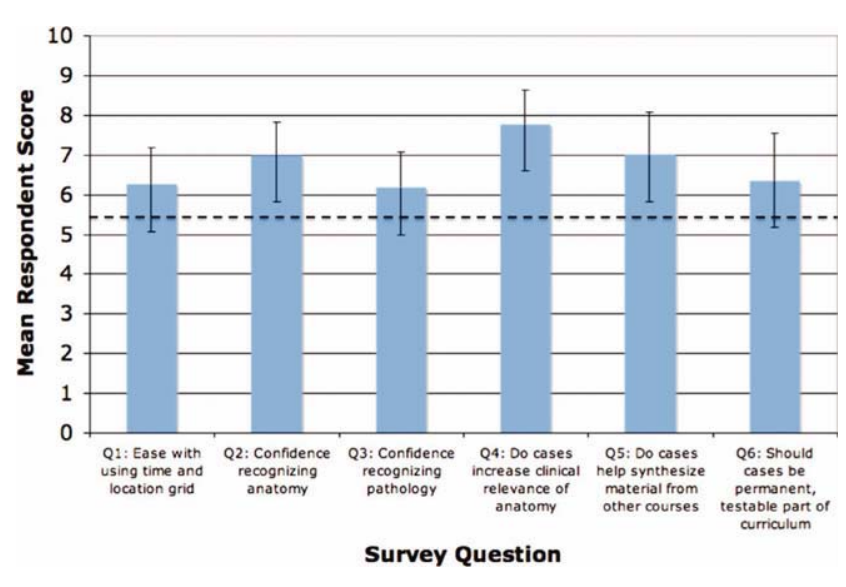

Dashed line indicates a neutral score of 5.5.

this feedback, we attempted to better integrate the pathology, pathophysiology, and pathohistology information with the radiological and anatomical considerations of the given disease process.

Two of the senior students had difficulty using the navigation grid. One student found it difficult to use the time scale at the bottom of the CT movie file, claiming, "I did not understand how to properly utilize the timing information provided in the paragraph describing the CT image." Another student thought the page layout could be improved, writing, "the grid info is not exactly easily put to memory while reading the instructions and would be helpful to see while reviewing the CT." We attempted to modify the clinical cases so that CT navigation directions and the CT movie files were as close to each other as possible, but we also recognized that the clinical case page layout will vary from student to student depending on the settings of the individual's web-browser. Several of the senior students also cited the importance of writing test questions based on the clinical cases if we want students to earnestly use them in their study of anatomy. As one senior student explains, "anatomy studying for me tended to be on high yield methods-things we were tested on... There are minimal CT scan questions on gross exams, that is why I don't say this would have been extremely helpful." Another student writes, "I would make [the clinical cases] testable so students will learn and devote effort into the CTs. I feel that most people will not look at it... because there many other things to require, but it would definitely be helpful if required." As we know from personal experience, students tend not to pay any attention to resources that are designated as supplementary, focusing instead only on what is designated as mandatory or "testable." Though test questions could not be written directly from the clinical cases for this year's freshman stu- dents, we are trying to make the clinical cases a "testable" resource for next year's incoming class.

\section{First-Year Student Feedback}

The current first-year medical students were emailed the survey a total of four times (once a week for 4 weeks) in order to increase the survey response rate. By the end of the fourth week new responses had stopped coming in. We received 69 responses from a class of 170 students, for a response rate of $41 \%$. Mean response scores to the six survey questions are summarized in Table 1 and the distributions of respondent scores are given for each question in Table 2. Most students felt that it was easy finding structures on the PMCT scans using our navigation grid (mean score $=6.26 / 10$ ), though $25 \%$ of students found using the alphanumeric grid moderately difficult (Score 4-5) and 8\% found it substantially difficult (score $<=3$ ). Students felt strongly that our clinical cases increased their confidence in finding anatomical structures on axial CT (mean score $=7.00 / 10$ ), with $38 \%$ of students reporting very strong improvement (score $>=8$ ) in their confidence. Students felt slightly less positive about the clinical cases' effectiveness in increasing their confidence recognizing common pathology on CT (mean score $=6.17 / 10$ ), though $73 \%$ of students still felt as though the cases positively affected them in this regard (score $>=6$ ). The large majority of students felt that our clinical cases increased the clinical relevance of their anatomy course (mean score $=7.77 / 10)$, with only $8 \%$ of students reporting a negative score (score $<=5$ ). Students also felt strongly that the clinical cases helped them to synthesize material they receive in numerous different classes (mean score $=7.01 / 10$ ), as $75 \%$ of students reported a positive score (score $>=6$ ) for this question. Finally, there was wide disagreement among students over whether these clinical cases should be included as a permanent, testable part of their anatomy course. The overall consensus was that clinical cases should be included as testable material (mean score $=6.36 / 10)$, though $34 \%$ of respondents felt otherwise (score $<=5$ ).

\section{DISCUSSION}

Though our navigation interface was positively assessed overall, a great deal of variability exists in student opinion of how easy it was to navigate the CT scans using our new interface. Student feedback in the survey comments ranged from some students thinking the "grid was very helpful," to others who wrote, "The grid system was really poor. Not very clear or reliable." The most frequent suggestion for improvement to the navigation interface was to include more labeled CT scans in order to ease the process of searching for certain findings. For example, one student wrote, "perhaps it would be possible to label one CT at key intervals throughout the scan so that we can double-check our understanding of the location of anatomical structures." Another student writes, "I think more still images of the CTs 


\begin{tabular}{|c|c|c|c|c|c|c|c|c|c|c|}
\hline \multirow[b]{2}{*}{ Survey Question } & \multicolumn{10}{|c|}{ Percentage of Students Responding with the Given Score } \\
\hline & $\begin{array}{l}\text { Definitely NO/ } \\
\text { Extremely } \\
\text { Difficult } \\
\end{array}$ & 2 & 3 & 4 & 5 & 6 & 7 & 8 & 9 & $\begin{array}{c}\text { Definitely Yes/ } \\
\text { Extremely } \\
\text { Easy }\end{array}$ \\
\hline $\begin{array}{l}\text { How easy was it finding } \\
\text { structures on the CT scan using } \\
\text { the time and location grid? }\end{array}$ & 1 & 1 & 6 & 4 & 21 & 19 & 23 & 14 & 7 & 3 \\
\hline $\begin{array}{l}\text { Do you feel more confident in } \\
\text { your ability to recognize } \\
\text { anatomical structures on cross- } \\
\text { sectional CT after reviewing } \\
\text { these clinical cases? }\end{array}$ & 0 & 0 & 1 & 10 & 4 & 19 & 29 & 19 & 13 & 6 \\
\hline $\begin{array}{l}\text { Do you feel more confident in } \\
\text { your ability to recognize } \\
\text { common pathologies on CT } \\
\text { scan after reviewing these } \\
\text { clinical cases? }\end{array}$ & 0 & 1 & 10 & 10 & 4 & 31 & 21 & 13 & 7 & 1 \\
\hline $\begin{array}{l}\text { Do you think the CT-based } \\
\text { clinical cases increase the } \\
\text { clinical relavance of the } \\
\text { anatomy taught in the } \\
\text { M1 year? }\end{array}$ & 1 & 0 & 0 & 3 & 4 & 10 & 21 & 26 & 16 & 19 \\
\hline $\begin{array}{l}\text { Do the CT-based clinical cases } \\
\text { help you to synthesize material } \\
\text { you receive in numerous } \\
\text { different classes (i.e., } \\
\text { pathology, physiology, } \\
\text { anatomy) into an overall picture } \\
\text { of disease presentation in a } \\
\text { patient, including diagnosis } \\
\text { via CT? }\end{array}$ & 0 & 3 & 6 & 3 & 13 & 13 & 21 & 14 & 13 & 14 \\
\hline $\begin{array}{l}\text { Do you think these CT-based clinical } \\
\text { cases should be included as a } \\
\text { permanent, testable part of } \\
\text { M1 anatomy? }\end{array}$ & I & 9 & 1 & 9 & 14 & 17 & 20 & 9 & 7 & 13 \\
\hline
\end{tabular}

with labels (arrows, etc.) pointing out the pathology would be helpful." Some also complained of the inexactness of the alphanumeric grid and the time bar, suggesting that we enable a "time point to time point [search] with arrow keys [to help] in locating specific structures." We believe the best improvement would be to add a dynamic feature to the clinical cases that would allow grid lines to appear over the CT scan whenever the student's mouse pointer is placed on the image. This would enable students to more confidently locate the designated anatomy or pathology, but still require them to explore the multiple cross-sections of the CT scan. Though we did include in some cases labeled screen shots of CT scans for findings that we thought may be difficult, we would like to avoid labeling all the findings on a CT scan because we believe the process of exploring the scans is important in developing confidence when viewing radiographic images, and that a great deal of learning occurs during the process of searching through cross-sections for a particular finding. It is therefore important to us to find the appropriate balance in our navigation interface of student guidance and freedom to discover.

Our results that show an increase in student confidence viewing radiological imaging after studying the clinical cases are very encouraging. We have found that one of the greatest challenges of using radiologi- cal imaging in anatomy education is overcoming low student confidence when approaching radiology. We were encouraged to find in our survey results evidence that use of the clinical cases not only moderated student anxiety over using radiology, but actually increased student interest in radiology and in the process of obtaining and reading a CT scan. One student wrote as a suggestion for improvement, "It would be really cool if we could 'play' with a CT machine and look at various sections of the human body." Another student suggested, "I think the ability to use the actual software that [UM Hospitals] use for image viewing would be awesome. That way we can get some practice with the software." We also found evidence in the student comments that an interactive learning tool, such as our clinical cases, may be a better method of learning for many students than a traditional lecture from a radiologist. One student writes, "I learned a lot more about CT imaging from these case studies than in our CT lecture... Being able to work through the scans and replay the movie was very helpful." These comments, along with our survey results, indicate that by providing students the appropriate balance of guidance and freedom for selflearning, student confidence can be increased to a level that enables them to get more out of radiological images used in their anatomy courses. Furthermore, the feedback we received from senior medical stu- 
dents who have progressed into their clinical training suggests that the use of our clinical cases may also enhance early student appreciation for the importance of studying anatomy via radiology, and may even lead to improved student performance reading patient images in the third and fourth years of medical school. We believe this would be an interesting direction for future research to take on the effectiveness of radiology-based clinical cases such as our own, as it would permit a controlled comparison of students who chose to use the CT-based clinical cases with those who did not. The study we present here lacks such a control group.

Another major challenge of using PMCT scans in anatomy education is getting scans of good enough quality that they can be used to teach first-year medical students. We found the quality of our pre-embalming CT scans to be quite good. In most PMCT scans, we had good resolution of soft tissue anatomy, including the abdominal vasculature down to the level of unnamed mesenteric branches and lower limb vasculature into the ankle. Our findings contribute to those of Chew et al. (2006) by showing that scanning cadavers prior to embalming can significantly improve the quality and educational utility of PMCT scans.

We believe that our survey results support the theory that PMCT-based clinical cases specific to students' assigned donors will increase student appreciation of the clinical relevance of anatomy, improve student understanding of cross-sectional anatomy on CT scan, and improve student ability to recognize common pathologies on CT scan. That students felt as though these clinical cases helped them synthesize material they have learned in numerous different classes also indicates that these clinical cases have value to students that extends beyond their anatomy curriculum. Not only did our senior medical student reviewers comment positively on the inclusion of histology, pathology, and physiology in the clinical cases, but several first-year students also indicated that they desired even more synthesis of other courses into the clinical cases. One student wrote, "The pictures below showing the histology, pathology, and gross anatomy with explanations are great. The overlap with path, histo, and gross anatomy is helpful, though more integration would be better." As Mitchell and Batty (2009) explain, the goal of first-year medical anatomy education should be to provide students a foundation for future learning and a platform for safe practice as an intern. If our intention is to provide students with foundational knowledge of structure in which to place their evergrowing knowledge of function, then it makes sense for us to also provide students with resources that demonstrate just how their functional knowledge rests on anatomical concepts. We believe our results support the conclusion that the clinical cases we have developed show students why the anatomy they are learning is foundational to their other medical courses, and that is why students so strongly reported an increase in their perception of anatomy's clinical relevance after viewing these cases. We believe that future development of our clinical cases should include more thorough integration of material from numerous other medical courses, and that future evaluation of these clinical cases should investigate whether their use improves student performance not only in radiology and anatomy, but in other medical science courses as well.

Anatomical education at the University of Michigan Medical School (UMMS) focuses on encouraging students to view their assigned donor as the first patient they will encounter in their medical training. As such, students can gain valuable insight into the clinical significance of gross anatomy by observing the medical histories of the cadavers they will dissect. The record of pathologies, variations, and surgical procedures found within their assigned bodies expose students to the breadth of medical knowledge they will acquire during their clinical training. It has recently been shown by Bergman et al. (2008) that student anatomy test performance relies in large part on teaching anatomy in a clinical context. Therefore, any method of teaching that places anatomy in a clinical context and increases student appreciation for anatomy's clinical relevance is likely to improve student acquisition of important anatomical concepts. Furthermore, demonstrating for students the CT appearance of pathologies found in the very cadavers they are dissecting will help students embrace the concept of approaching the cadaver as though he or she is their first patient. Preclinical medical students are often eager to embrace the role of doctor and to learn clinically relevant material, and as Moxham and Plasiant (2007) show, "[students] at all stages of their medical course share with professional anatomists the view that anatomy is a very important subject for their clinical studies." Our results show that PMCT scans of cadavers can encourage this trend in student opinion; while at the same time enhance respect and empathy for the donor by bringing the donor's identity as a patient back to life. Although Lufler et al. (2010) show that student use of radiographic images not specific to the body they are dissecting can be valuable, it is our personal experience that cadaverspecific images generate tremendous interest among students in both the images and in the bodies. We also believe that by providing students with images specific to their assigned bodies, we encourage students to assume the role and professional behavior of a physician, and we promote student respect for donors by treating donors as patients.

We also recognize, however, that our gratis arrangement with the UM Radiology Department is a privilege not all medical schools may have, and that the cost of scanning bodies each year for student dissection is prohibitive. For those schools that wish to employ radiology-based clinical cases into their anatomy curricula but lack the resources to obtain bodyspecific images, we believe a standard set of CT scans could serve as a substitute if the scans were matched to cadavers that are known to exhibit pathologies present in the scans. Such a project would necessitate collecting patient histories for the donors and searching through them for disease processes that would appear on a CT scan. Although this would require a substantial amount of work to prepare for each year's 
dissection course, it is our experience that such a project is ideal for medical or premedical students looking for a summer project. We have also found that firstyear medical students really value having the anonymized medical histories of their donors and that providing students with donor histories further encourages student respect for the donor as a person.

As modern medical school curricula evolve, many medical schools continue to truncate or altogether remove dissection from their anatomy curricula, turning to web-based learning (computer-aided instruction, CAI) and radiological imaging as replacements for dissection rather than supplements to it. A large body of research indicates that both anatomy professors and students believe CAI and radiological imaging are most useful when used in combination with dissection (Erkonen et al., 1992a,b; Holla et al., 1999; Aziz et al., 2002; Rizzolo et al., 2002; Jastrow and Vollrath, 2003; Gunderman and Wilson, 2005; Miles, 2005; Chew et al., 2006; Azer and Eizenberg, 2007; Turney, 2007; Petersson et al., 2009). Evidence supporting the validity of this opinion can be found in the study by Erkonen et al. (1992a,b), which sought to determine whether ultra-fast CT videotape of the heart could enhance or substitute for cadaver dissection in teaching first-year medical students anatomy. The results showed that CT imageacquired knowledge did not transfer to cardiac specimen identification, and so the ultra-fast CT videotape was not a suitable substitute for cadaver dissection. Furthermore, a recent study comparing student CAI usage and test-performance produced results that suggest student reliance on CAI as a primary learning method, rather than supplementary, results in sub-average test scores (Rizzolo et al., 2002). Although financial constraints and curricular changes have pushed medical institutions to try to supplant dissection with CAI and radiology, the above evidence, in addition to the sentiment of the majority of students and professors, suggests that traditional cadaver dissection is an essential component of anatomy education (Aziz et al., 2002; Chew et al., 2006; Azer and Eizenberg, 2007). Miles (2005) wrote, "although more research is needed in this area, current evidence suggests that imaging and cadaveric dissection may be complementary rather than competitive tools for learning anatomy." Our results support the notion that CAI and radiology can be great complements to cadaver dissection, and we would recommend the use of clinical cases similar to those we present here to any medical school utilizing student cadaver dissection as a primary teaching method in anatomy education.

\section{REFERENCES}

Azer S, Eizenberg N. 2007. Do we need dissection in an integrated problem-based learning medical course? Perceptions of firstand second-year students. Surg Radiol Anat 29:173-180.
Aziz MA, McKenzie JC, Wilson JS, Cowie RJ, Ayeni SA, Dunn BK. 2002. The human cadaver in the age of biomedical informatics. Anat Rec 269:20-32.

Bergman EM, Prince KJ, Drukker J, van der Vleuten CP, Scherpbier AJ. 2008. How much anatomy is enough? Anat Sci Educ 1:184-188.

Chew FS, Relyea-Chew A, Ochoa ER Jr. 2006. Postmortem computed tomography of cadavers embalmed for use in teaching gross anatomy. J Comput Assist Tomogr 30:949-954.

de Barros N, Rodrigues CJ, Rodrigues AJ Jr, de Negri Germano MA Cerri GG. 2001. The value of teaching sectional anatomy to improve CT scan interpretation. Clin Anat 14:36-41.

Erkonen WE, Albanese MA, Smith WL, Pantazis NJ. 1990. Gross anatomy instruction with diagnostic images. Invest Radiol 25:292294.

Erkonen WE, Albanese MA, Smith WL, Pantazis NJ. 1992a. Effectiveness of teaching radiologic image interpretation in gross anatomy. A long-term follow-up. Invest Radiol 27:264-266.

Erkonen WE, Krachmer M, Cassell MD, Albanese MA, Stanford W. 1992b. Cardiac anatomy instruction by ultrafast computed tomography versus cadaver dissection. Invest Radiol 27:744-747.

Forrester D. 1971. Teaching anatomy through radiology. A new challenge requiring new techniques. Radiology 100:561-565.

Gunderman RB, Wilson PK. 2005. Viewpoint: Exploring the human interior: The roles of cadaver dissection and radiologic imaging in teaching anatomy. Acad Med 80:745-749.

Holla SJ, Selvaraj KG, Isaac B, Chandi G. 1999. Significance of the role of self-study and group discussion. Clin Anat 12:277-280.

Jacobson S, Epstein SK, Albright S, Ochieng J, Griffiths J, Coppersmith V, Polak JF. 2009. Creation of virtual patients from CT images of cadavers to enhance integration of clinical and basic science student learning in anatomy. Med Teach 31:749-751.

Jastrow H, Vollrath L. 2003. Teaching and learning gross anatomy using modern electronic media based on the visible human project. Clin Anat 16:44-54.

Khalil MK, Payer AF, Johnson TE. 2005. Effectiveness of using crosssections in the recognition of anatomical structures in radiological images. Anat Rec B New Anat 283:9-13.

Lufler RS, Zumwalt AC, Romney CA, Hoagland TM. 2010. Incorporating radiology into medical gross anatomy: Does the use of cadaver CT scans improve students' academic performance in anatomy? Anat Sci Educ 3:56-63.

McNiesh LM, Madewell JE, Allman RM. 1983. Cadaver radiography in the teaching of gross anatomy. Radiology 148:73-74.

Miles KA. 2005. Diagnostic imaging in undergraduate medical education: An expanding role. Clin Radiol 60:742-745.

Mitchell R, Batty L. 2009. Undergraduate perspectives on the teaching and learning of anatomy. ANZ J Surg 79:118-121.

Moxham BJ, Plaisant O. 2007. Perception of medical students towards the clinical relevance of anatomy. Clin Anat 20:560-564.

Pantoja E, Nagy F, Zambernard J. 1985. Clinical radiographs of the cadaver as a teaching aid in anatomy. Radiology 155:28.

Petersson H, Sinkvist D, Wang C, Smedby O. 2009. Web-based interactive 3D visualization as a tool for improved anatomy learning. Anat Sci Educ 2:61-68.

Rizzolo LJ, Aden M, Stewart WB. 2002. Correlation of Web usage and exam performance in a human anatomy and development course. Clin Anat 15:351-355.

Stassa G, Evans JA. 1969. Radiology, anatomy, and the medical student. Radiology 92:1562-1563.

Tavares MA, Dinis-Machado J, Silva MC. 2000. Computer-based sessions in radiological anatomy: One year's experience in clinical anatomy. Surg Radiol Anat 22:29-34.

Turmezei TD, Tam MD, Loughna S. 2009. A survey of medical students on the impact of a new digital imaging library in the dissection room. Clin Anat 22:761-769.

Turney BW. 2007. Anatomy in a modern medical curriculum. Ann R Coll Surg Engl 89:104-107. 